www.jmscr.igmpublication.org

Impact Factor (SJIF): 6.379

Index Copernicus Value: 71.58

ISSN (e)-2347-176x ISSN (p) 2455-0450

crossref DOI: _https://dx.doi.org/10.18535/jmscr/v6i4.40

Journal Of Medical Science And Clinical Research

\title{
A Comparative Study of Visual Field Defects in Normal-Tension and High-Tension Glaucoma
}

\author{
Authors \\ Dr Manushree Gautam, Dr Parul Malviya, Dr Preeti Rawat \\ MGM Medical College
}

\begin{abstract}
Objective: observing difference in pattern of disc and visual field damage produced by NTG and HTG.

Methodology: A Cross sectional study conducted at MYH \& MGM Medical College, Indore, from Oct 2015 to oct 2017. The visual fields showing defects suggestive of glaucoma were taken for study and 22 such fields of 22 eyes with normal-tension glaucoma were compared to 20 visual fields of 20 eyes showing high-tension glaucoma. We studied the differences in the number of patients with defects extending to within 10 degrees of central fixation, in the amount of localized visual field loss (pattern standard deviation), and in the amounts of generalized visual field loss (mean deviation) in normaltension glaucoma and high-tension glaucoma groups.

Results: During the study period, 42 eyes of 42 patients were taken up for this cross-sectional analysis including 22 NTG and 20 HTG patients were analysed. Out of total 42 patients, 24(57.14\%) were males and $18(42.86 \%)$ were females. The mean age of the patients was $31 \pm 4.6$ years in NTG and $39 \pm 2.8$ years in HTG groups. We also found characteristic differences in field defects among NTG and HTG groups in most of our patients where the NTG patients had a greater amount of focal visual field loss (pattern standard deviation) and greater paracentral field defects.
\end{abstract}

Conclusion: In conclusion our study suggests that the visual fields defects of NTG patients differ from that of HTG patients. As field defects of NTG patients affect the central vision earlier, more vigilant and frequent monitoring is required in these cases.

\section{Introduction}

Normal tension or low tension glaucoma is a variety of POAG that features an intraocular pressure within the normal range. Total population surveys show that $10-30 \%$ of patients newly diagnosed with glaucoma have IOPs that are and remain normal $(<21 \mathrm{mmHg})$. Interestingly, in Japan, $50-60 \%$ of patients affected by open-angle glaucoma have baseline IOPs below this level. The traditional therapy for primary open-angle glaucoma is to lower IOP to within the normal range, but this approach becomes more difficult when the initial IOP is "normal". The pathogenesis of the condition remains unclear, although vascular insufficiency, nocturnal hypotension and peripheral vasospasm are suggested. The association with migraine, and recurrent optic disc hemorrhages supports this hypothesis. The most effective management has been to lower IOP. Color Doppler imaging has demonstrated an increase in blood velocity in the ophthalmic artery after filtration surgery which suggests a mechanical hypothesis in which the IOP is "too high for the eye," and the optic nerve 
is less able to withstand an IOP in the normal range. Many researchers, since the first report in 1857 by Von Graefe, have studied the structural and functional differences between NTG and POAG. The targets were optic nerve head, visual field, and RNFL.

- Visual field defects of NTG and POAG have been analyzed in many studies, in which some authors reported no differences between NTG and POAG while others concluded that the visual field defect in NTG was characteristically different from that of POAG.

- The purpose of our study is to confirm the hypothesis that pathogenesis of normaltension glaucoma differs from that of hightension glaucoma by the means of observing difference in pattern of disc and visual field damage produced by both the entities.

\section{Materials and method}

- A Cross sectional study conducted at MYH \& MGM Medical College, Indore, from Oct 2015 to oct 2017.

- Written informed consent to participate in the study was obtained from all patients or from parents of minor subjects.

We defined NTG according to the following criteria:

- Maximum recorded IOP less than $21 \mathrm{mmHg}$ without antiglaucoma medication,

- Characteristic glaucomatous optic nerve head changes,

- Glaucomatous visual field loss,

- open anterior chamber angle in all quadrants

- POAG was defined to meet the same criteria of NTG except for intraocular pressure $>25 \mathrm{~mm} \mathrm{Hg}$ in an eye without antiglaucoma medication. Complete ophthalmological examination including IOP measurement done using Goldmanns applanation tonometer which was corrected for central corneal thickness, taking mean value as $540 \mu$.

- Optic disc examination done by using a slit-lamp biomicroscope and a Volk 78D double aspheric lens.

Disc changes were considered to be glaucomatous if they met the following criteria -

1) Vertical cup-disc ratio $>0.5: 1$

2) Asymmetry between cup size of both eyes $>2 \mathrm{~mm}$

3) Optic disc showing NRR thinning or notching/ NRR pallor/laminar dot sign/ bayonetting of vessels/disc hemorrhage.

\section{Inclusion Criteria}

- Age group 10-60 yrs.

- Refraction $+3 \mathrm{D}$ to $-4 \mathrm{D}$

- $\mathrm{IOP}<21 \mathrm{mmHg}$ (NTG group)

- a pupil diameter of at least $3 \mathrm{~mm}$

- A reliable visual field test

(fixation losses less than 20\% and falsepositive and false-negative errors less than $33 \%$ )

\section{Exclusion Criteria}

- $\mathrm{MD}$ (mean deviation) of more than $-14 \mathrm{~dB}$ on Humphrey C30-2 program.

By this we excluded the eyes with severe visual field defects from this study

- Patients with any ocular diseases known to affect the visual field, such as diabetic retinopathy, neurological diseases, macular degeneration or a vascular occlusion.

- Myopia > 4D

- Other ocular pathologies such as retinal diseases and cataract.

Automated perimetry was performed with Humphrey visual field analyzer Zeiss II (Central 30-2 Threshold Program using Stimulus III) .The results were classified to be glaucomatous changes according to Modified Anderson's criteria. The visual fields showing defects suggestive of glaucoma were taken for study and 22 such fields of 22 eyes with normal-tension glaucoma were compared to 20 visual fields of 20 eyes showing hightension glaucoma. 
- Patients of NTG and HTG were closely matched for the age, sex and stage of glaucoma as the maximum allowable difference in mean deviation was kept 0.3 $\mathrm{dB}$ to determine any differences in the characteristics of visual field defects between the two groups.

- We studied the differences in the number of patients with defects extending to within 10 degrees of central fixation, in the amount of localized visual field loss (pattern standard deviation), and in the amounts of generalized visual field loss (mean deviation) in normal-tension glaucoma and high-tension glaucoma groups.

\section{Results}

During the study period, 42 eyes of 42 patients were taken up for this cross-sectional analysis including 22 NTG and 20 HTG patients were analysed. Out of total 42 patients, 24(57.14\%) were males and $18(42.86 \%)$ were females. The mean age of the patients was $31 \pm 4.6$ years in NTG and $39 \pm 2.8$ years in HTG groups.

\begin{tabular}{|l|c|c|}
\hline Age group & NTG & HTG \\
\hline $11-20$ yrs & 2 & 0 \\
\hline $21-30$ yrs & 4 & 2 \\
\hline $31-40$ yrs & 8 & 7 \\
\hline $41-50$ yrs & 5 & 8 \\
\hline $51-60$ yrs & 3 & 3 \\
\hline
\end{tabular}

We also found characteristic differences in field defects among NTG and HTG groups in most of our patients where the NTG patients had a greater amount of focal visual field loss (pattern standard deviation) which can be explained in Table-2

\begin{tabular}{|l|c|c|}
\hline & $\begin{array}{c}\text { NTG group } \\
(22 \text { patients })\end{array}$ & $\begin{array}{c}\text { HTG group } \\
(\mathbf{2 0} \text { patients) }\end{array}$ \\
\hline MD & $-5.32 \mathrm{~dB}$ & $-5.18 \mathrm{~dB}$ \\
\hline PSD & $6.47 \mathrm{~dB}$ & $5.25 \mathrm{~dB}$ \\
\hline Focal defects & $14(63 \%)$ & $11(55 \%)$ \\
\hline Superior field defects & $5(35 \%)$ & $5(45 \%)$ \\
\hline Inferior field defects & $9(64 \%)$ & $6(54 \%)$ \\
\hline
\end{tabular}

\section{Discussion}

- Many researchers have studied the structural and functional differences between NTG and POAG. The targets were optic nerve head, visual field, and RNFL.

- Visual field defects of NTG and POAG have been analysed in many studies, in which some authors reported no differences between NTG and POAG while others concluded that the visual field defect in NTG was characteristically different from that of POAG.

- The significant evidence of differences in NTG and POAG would imply pathogenic differences of optic nerve damage between NTG and POAG.

- Caprioli et al reported that the visual field defects in normal tension glaucoma occurred closer to fixation and that the slope and depth of the scotoma was steeper and greater in low tension glaucoma than those in high tension glaucoma.

They suggested that in high tension glaucoma, the progression of visual field loss started from a more peripheral toward a more central field, while in low tension glaucoma, the visual field loss was very close to fixation and the pattern of progression was not related to the thickness of the neural rim.

- Disproportionately greater cupping compared with comparable visual field loss was reported in patients with NTG. Caprioli et al also suggested that significant correlation existed between the thickness of the neural rim and the distance of the deepest scotoma from fixation only in high tension glaucoma, but not in low tension glaucoma.

- Recently, Chauhan and associates found that individuals with normal-tension glaucoma had greater areas of normal sensitivity and, therefore, more localized visual field defects than individuals with high-tension glaucoma. 
- We also found characteristic differences in field defects among NTG and HTG groups in most of our patients where the NTG patients had a greater amount of focal visual field loss (pattern standard deviation) Previously, two separate studies had shown that patients with glaucoma with generalized visual field loss had a higher mean intraocular pressure than those with more localized visual field loss.

- Paul, Cohn, and Weber who recently found no significant difference in the pattern standard deviation between patients with low tension glaucoma (intraocular pressure $<21 \mathrm{~mm} \mathrm{Hg}$ ) and patients with hightension glaucoma (intraocular pressure $>28 \mathrm{~mm} \mathrm{Hg}$ ) with similar mean deviations.

\section{Conclusion}

- In conclusion our study suggests that the visual fields defects of NTG patients differ from that of HTG patients

- This can aid into the hypothesis that the pathogenesis of both glaucomas varies hence the treatment preferrence will be given to the antiglaucoma medications which have neuroprotective action by increasing the vascular supply of optic nerve.

- As field defects of NTG patients affect the central vision earlier, more vigilant and frequent monitoring is required in these cases.

\section{References}

1. Caprioli J, Spaeth GL. Comparison of visual field defects in the low-tension glaucomas with those in the high-tension glaucomas. Am J Ophthalmol 1984;97: 730-7.

2. Lewis RA, Heyreh SS, Phelps CD. Optic disk and visual field correlations in primary open angle and low-tension

\section{glaucoma. Am J Ophthalmol 1983;96:148-52.}

3. Caprioli J, Spaeth GL. Comparison of the optic nerve head in high- and low-tension glaucoma. Arch Ophthalmol 1985;130: 1145-9.

4. John H. Zeiter at al Am J Ophthalmology 114:758-763, DECEMBER, 1992

5. Ariae at al Visual Field Defects in Normal tension and High tension Glaucoma by Makoto Araie, MD, Junkichi Yamagami, MD, Yasuyuki Suziki, MD

6. 6.Collaborative Normal Tension Glaucoma Study Group. (1998). The Effectiveness of Intraocular Pressure Reduction in the Treatment of Normal Tension Glaucoma.American Journal of Ophthalmology, Vol.126, pp. 498-505

7. Shields, M.B. (2008). Normal-Tension Glaucoma: Is It Different From Primary Open-Angle Glaucoma? Current Opinion in Ophthalmology, Vol. 19, No. 2, p. 85-88

8. Comparison of localised nerve fibre layer defects in normal tension glaucoma and primary open angle glaucoma S J Woo, K H Park, D M Kim Br J Ophthalmol 2003;87:695-698

9. Chauhan BC, Drance SM, Douglas GR, Johnson CA. Visual field damage in normal-tension and high-tension glaucoma. Am J Ophthalmol 1989; 108:636-42.

10. ouri-Mahdavi K, Hoffman D, Gaasterland D, Caprioli J. Prediction of visual field progression in glaucoma. Invest Ophthalmol Vis Sci 2004; 45(12): 4346-4351. 